\title{
Historical correlates of genital herpes simplex virus type 2 infection in men attending an STD clinic
}

\author{
J M Sizemore Jr, F Lakeman, R Whitley, A Hughes, E W Hook III
}

Sex Transm Infect 2005;81:303-305. doi: 10.1136/sti.2004.011957

Background/objectives: While nearly one in four Americans has antibodies to HSV-2, only one of 40 reports a history of genital herpes $(\mathrm{GH})$. The goal of this study was to correlate questions designed to elicit a GH history with serological evidence of HSV-2 in male STD clinic attendees.

Methods: Consecutive males were enrolled in a study of the epidemiology of $\mathrm{GH}$. Consenting men answered questionnaires regarding their histories of possible $\mathrm{GH}$ and underwent serological testing for HSV-1 and HSV-2. Association statistics between response to each question and HSV-2 serological status were calculated.

Results: Of 328 men enrolled, 148 (46\%) had HSV-2 antibodies. $14(4.3 \%)$ reported a history of $\mathrm{GH}$ when queried as part of a list of other STD (sensitivity (S) 0.08). 17 (5.2\%) reported a history of GH when asked directly "Do you have genital herpes?" (S 0.09). 75 (21.1\%) participants reported a history of a recurring genital sore, ulcer, or zipper cut (S 0.32 ). Overall, $64.2 \%$ of HSV-2 seropositive men answered "no" to all three questions. A "yes" response to any of the questions was only $36 \%$ sensitive for predicting HSV-2 infection.

Conclusion: Few HSV-2 infected men report either a history of $\mathrm{GH}$ or are aware that they are infected. Asking about a history of recurrent genital sores was a more sensitive historical marker of HSV-2 infection than asking about a history of "genital herpes."

n the United States, one in four adults is infected with herpes simplex virus type 2 (HSV-2). Most, however, do not recognise this infection; in the National Health and Nutrition Examination Survey (NHANES) III, while one in 40 participants reported a history of genital herpes $(\mathrm{GH})$, only one in 10 of those infected stated they had GH. Furthermore, only one in 30 black people, a population with substantially higher HSV-2 prevalence, reported a history of GH.'

A published study from Seattle suggests that three quarters of infected individuals who do not report a history of GH actually have unrecognised infection, not truly asymptomatic infection. ${ }^{2}$

To further address patient recognition of HSV-2 infections, we examined historical correlates of HSV-2 among men attending an STD clinic in the south eastern United States, a previously understudied population.

\section{METHODS}

\section{Study population}

The study was conducted at the Jefferson County Department of Health STD Clinic in Birmingham, AL. Consecutive heterosexual men presenting to the clinic were approached for enrolment.
This study was reviewed and approved by the institutional review board for human subjects at the University of Alabama at Birmingham.

\section{Study procedures}

After providing written informed consent, participants were administered a questionnaire collecting demographic data, STD history, sexual partner history, and information on GH knowledge.

Included were three questions targeting a history of GH. Firstly, participants were asked if they had ever had GH among a list of STDs; secondly, in a different section assessing knowledge about herpes, subjects were asked, "Do you have genital herpes?" Finally, men were later queried, "Have you ever had a recurring genital sore, ulcer, or zipper cut on your penis, scrotum, or anus?"

Participants then underwent a physical examination. Urine specimens for cervical gonorrhoea and Chlamydia trachomatis were collected; serum was obtained for VDRL, HIV, and HSV serological testing. Participants then received care per clinic protocol.

\section{Herpes laboratory procedures}

Serological testing for HSV-1 and HSV-2 was conducted in the laboratory of RW and FL using Herpeselect- 1 and Herpeselect-2 ELISA IgG (98\% sensitivity, 96\% specificity, Focus Technologies, Inc, Herndon, VA, USA).

\section{Data management and statistical analysis}

Questionnaire and laboratory data were entered into a Microsoft Access database with appropriate checks to ensure accuracy.

Analyses were conducted with SAS software (SAS Institute, Cary, NC, USA). Odds ratios were calculated using Fisher's exact test.

\section{RESULTS}

\section{Study population}

Between February 2002 and September 2003, 328 men were enrolled. The majority were young (median age, 26 years) and black $(90 \%)$. Reasons for clinic visit included symptoms $(52.3 \%)$, check up $(30.3 \%)$, STD contact $(16.2 \%)$, and other (1.2\%).

These men had a substantial duration of sexual experience (median 11 years) defined as their age of sexual debut (median 15 years) subtracted from their current age. Participants reported a median of one partner in the last 30 days and 16 lifetime partners.

At the study visit, $45 \%$ (148/328) of men were HSV-2 seropositive, while $62.2 \%$ were HSV-1 seropositive.

When asked if they had GH among a list of STDs, 4.3\% (14/ 328 ) of the overall population and $8.1 \%$ (12/148) of HSV-2 seropositive men answered affirmatively (odds ratio (OR)

Abbreviations: $\mathrm{GH}$, genital herpes; HSV, herpes simplex virus 
Table 1 Comparison of genital herpes historical correlates among 328 male STD clinic attendees

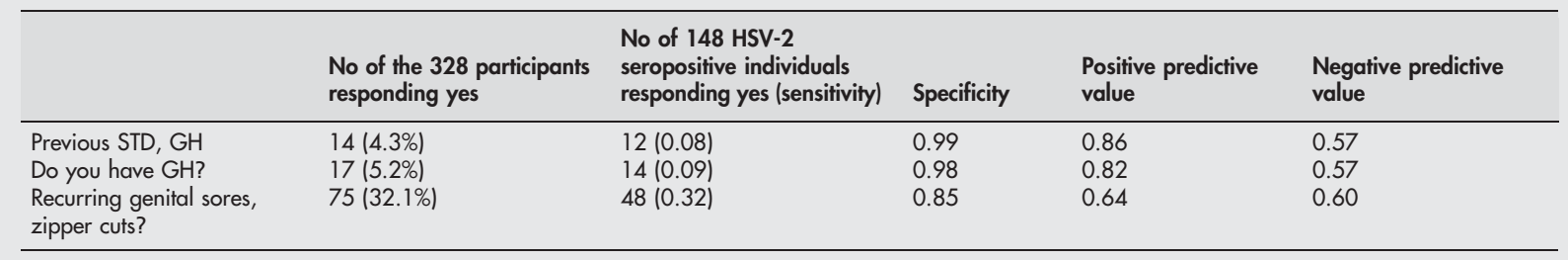

7.8, $\mathrm{p}=0.0018)$. Similarly, 5.2\% (17/328) of all participants and $9.5 \%(14 / 148)$ of those with HSV-2 infection answered "yes" when asked directly, "Do you have genital herpes?" (OR 6.2, $\mathrm{p}=0.0015$ ). Ten of the 21 participants responding "yes" to one of the questions answered "yes" to both questions. Finally, 23\% (75/328) of all men and 32.4\% (48/ 148) of HSV-2 infected men reported a history of previous recurrent genital ulcer, sore, or zipper cut (OR 2.7, $\mathrm{p}=0.0002$ ) (table 1). Sixty four per cent $(\mathrm{n}=95)$ of HSV-2 seropositive men answered "no" to all three questions; a "yes" response to any of the three questions was 36\% (53/ 148) sensitive for predicting HSV-2 infection. There was no difference in herpes historical accounts among those infected with HSV-2 when stratified by HSV-1 serological status $(\mathrm{p}=0.35)$.

\section{DISCUSSION}

The sensitivity of reporting a history of GH for predicting HSV-2 infection as measured by antibody is uniformly low in both general and high risk populations. When asked about a history of GH among a list of STD, our findings were similar to those reported from NHANES III. The sensitivity of a self reported history of $\mathrm{GH}$ in that study for the general population and for black people was $9.2 \%$ and $3.7 \%$, respectively ${ }^{1}$; it was also noted that those who were HSV-2 seropositive only were three times more likely to report a history of GH (16.2\%) than individuals seropositive for HSV-1 and HSV-2 (5.6\%), ${ }^{3}$ suggesting that those co-infected with HSV-1 may have been less symptomatic. Similarly, in another study conducted among randomly selected attendees of primary care offices of six US cities, Leone et al report that one in four individuals were infected with HSV-2, though only $4 \%$ disclosed a history of GH; there, the sensitivity of reporting a GH history for predicting presence of HSV-2 antibody was $11.9 \%{ }^{4}$

Previous studies addressing self recognition of HSV-2 infection in high risk individuals are few. One study that examined self reported GH history in the context of screening was a secondary cross sectional analysis from Project Respect, a randomised trial of STD prevention programmes among STD clinic attendees. Of 4128 individuals attending five US STD clinics, $40.8 \%$ (1686/4128) were HSV-2 seropositive; among these only $11.7 \%$ reported a history of $\mathrm{GH}$. Participants who were black, female, or HSV-l seropositive were more likely to have unrecognised $\mathrm{GH} .^{5}$

Our findings regarding heterosexual high risk men confirm as well as add new insights to these published data. Firstly, genital HSV-2 infection was common and poorly recognised. However, when questioned using different queries to elicit a history of possible GH, this predominantly black STD clinic population recognised infection three times more commonly than reported in NHANES III. Asking about recurring genital lesions was a more sensitive historical marker for HSV-2 infection than asking directly about genital herpes, suggesting that a substantial proportion of these men recognise abnormalities but do not associate them with GH. Finally, while the proportion of those seropositive for HSV-1 infection was not different from those of NHANES III and Project Respect, we did not identify HSV-1 serostatus as a modifying factor in the recognition of genital HSV-2 infection as had been reported previously.

Several limitations of this study should be acknowledged and considered. Firstly, these data were collected from a single STD clinic in the south eastern United States, and, thus, may not be generalisable to other populations. None the less, we have described participants from the racial ethnic group (black people) in whom HSV-2 seroprevalence is highest, recruited from the region (southeastern United States) where infection is more common. ${ }^{1}$ Finally, a few responses from those who have genital HSV-1 disease could have been misclassified, though we have found little evidence of HSV-1 genital infection in this population.

\section{CONCLUSION}

Though HSV-2 is common, few men report a history of GH. Asking about a history of recurrent genital sores was a more sensitive marker of HSV-2 infection than asking about a history of GH, though most did not recognise the former fact as evidence of infection. Antibody screening and patient education about manifestations of genital herpes should be emphasised as important prevention tools against the spread of HSV-2 among high risk heterosexual males.

\section{ACKNOWLEDGEMENT}

Special thanks to Marga Jones for assistance with data management.

\section{CONTRIBUTORS}

JS, protocol development, participant enrolment, manuscript preparation, revision; FL, herpes laboratory testing, manuscript review; RW, protocol development, herpes laboratory testing, manuscript review; AH, participant enrolment, manuscript review; EH, protocol development, non-herpes STD laboratory testing, manuscript review, revision.

\section{Authors' affiliations}

J M Sizemore Jr, A Hughes, E W Hook III, Division of Infectious Diseases, University of Alabama at Birmingham, Birmingham, AL, USA F Lakeman, R Whitley, Division of Pediatric Infectious Diseases, University of Alabama at Birmingham, Birmingham, AL, USA

E W Hook III, Jefferson County Department of Health, Birmingham, AL, USA

Grant support: Supported by the UAB Sexually Transmitted Diseases Cooperative Research Center Grant 5 U19 Al38514-07, the UAB GCRC, RR00032.

No conflict of interest for any of the authors.

Correspondence to: James M Sizemore Jr, The University of Alabama at Birmingham 703 19th Street South, 242 Zeigler Research Building Birmingham, AL 35294-0007, USA; sizemore@uab.edu

Accepted for publication 1 November 2004

\section{REFERENCES}

1 Fleming DT, McQuillan GM, Johnson RE, et al. Herpes simplex virus type 2 in the United States, 1976 to 1994. N Engl J Med Oct 16, 1997;337:1 105-11. 
2 Corey L. The current trend in genital herpes. Progress in prevention. Sex Transm Dis 1994;21(Suppl):S38-44.

3 Xu F, Schillinger JA, Sternberg MR, et al. Seroprevalence and coinfection with herpes simplex virus type 1 and type 2 in the United States, 1988-1994. J Infect Dis 2002;185: 1019-24.
4 Leone $\mathbf{P}$, Fleming DT, Gilseman AW et al. Seroprevalence of herpes simplex virus -2 in suburban primary care offices in the United States. Sex Transm Dis virus -2 in suburban

5 Gottlieb S, Douglas J, Schmid D, et al. Seroprevalence and correlates of herpes simplex virus type 2 infection in five sexually transmitted diseases clinics. J Infect Dis 2002;186:1381-9.

$\mathrm{ECHO}$

\section{Antiretroviral drugs confuse universal antenatal blood screens}

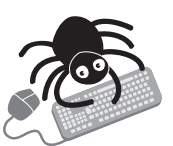

Please visit the Sexually Transmitted Infections website [www. stiijournal.com] for a link to the full text of this article.
Il women infected with HIV should have their haemoglobin $\mathrm{A}_{2}\left(\mathrm{HbA}_{2}\right)$ concentrations $\triangle$ recorded before they start antiretroviral drug treatment to avoid later suspicion Iantenatally of heterozygous $\beta$ thalassaemia trait and unnecessary genetic counselling and analysis, say some UK doctors. In their experience $\mathrm{HbA}_{2}$ concentration can be raised solely by the treatment.

This is likely to become more of a problem now that universal antenatal screening for blood disorders is to be introduced to areas of the UK where $\beta$ thalassaemia is prevalent and because more and more fertile women are being treated with antiretroviral drugs for HIV infection. A benchmark $\mathrm{HbA}_{2}$ value will allow doctors to judge whether to exclude heterozygous $\beta$ thalassaemia.

The doctors reported on two cases in which pregnant women being treated with antiretroviral drugs had raised $\mathrm{HbA}_{2}$ concentrations which were not associated with hypochromic, microcytic red cell indices characteristic of heterozygous $\beta$ thalassaemia. One was in a 35 year old with asymptomatic HIV infection and hepatitis C infection since 1990, receiving antiretroviral drugs since 1992. Stavudine, didanosine, and nevirapine were changed to zidovudine, lamivudine, and efavirenz in 2000 and efavirenz to nelfinavir later, when she was eight weeks pregnant, to avoid teratogenic effects. Hb concentration was $124 \mathrm{~g} / \mathrm{l}, \mathrm{HbA} 95.7 \%$, and $\mathrm{HbA}_{2} 4.3 \%$ on booking in to the antenatal clinic. This led to her and her partner's referral to counsellors-as if she were positive for heterozygous $\beta$ thalassaemia-and testing of her partner, who was HIV positive but taking no antiretroviral drugs: $\mathrm{Hb}$ was $118 \mathrm{~g} / \mathrm{l}, \mathrm{HbA} 87.2 \%$, and $\mathrm{HbA}_{2} 6.3 \%$. Genetic analysis on her partner and to ascertain her carrier status for $\beta$ thalassaemia and risk to the fetus showed that he had heterozygous $\beta$ thalassaemia; sequencing showed no mutation in her $\beta$ globlin gene. Healthy twins were born at term with normal results for full blood counts and on neonatal haemoglobinopathy screening.

The other case was in a 32 year old whose haemoglobin concentration was $90 \mathrm{~g} / \mathrm{l}, \mathrm{HbA}_{2}$ $1.6 \%$ in 1996 but who later contracted AIDS and in 1999 started receiving antiretroviral drugs. In early 2000 she was taking zidovudine, lamivudine, and nelfinavir; zidovudine was later replaced by stavudine because of severe anaemia. Later in 2000, when she was pregnant, blood tests at booking in disclosed an $\mathrm{HbA}_{2}$ concentration of $3.9 \%$, even though she had stopped zidovudine 10 weeks before. After counselling, blood tests confirmed that her partner did not have $\beta$ thalassaemia. Two years later nelfinavir was changed to nevirapine to ensure compliance, and three months afterwards, during her second pregnancy, $\mathrm{HbA}_{2}$ concentration was $2.9 \%$.

Zidovudine has been reported to raise $\mathrm{HbA}_{2}$ concentrations, and it seemed to do so in case 1. A similar effect may have been prolonged in case 2 because of the long half life of erythrocytes- 120 days. $\mathrm{HbA}_{2}$ concentration was normal two years later, without zidovudine, though stopping nelfinavir cannot be excluded as an alternative explanation for this.

Heterozygous $\beta$ thalassaemia, which raises $\mathrm{HbA}_{2}$ concentrations to $4-6 \%$, is important in antenatal screening for blood disorders and shows hypochromic, microcytic red cell indices, but is not to be confused with other conditions with raised $\mathrm{HbA}_{2}$ concentrations, like hyperthyroidism and megaloblastic anaemia or being HIV positive and taking antiretroviral drugs, when these markers are absent. Previous, selective antenatal screening of $\mathrm{HbA}_{2}$ has been applied only when the characteristic markers of $\beta$ thalassaemia are present or suspect. However, as these cases show, raised $\mathrm{HbA}_{2}$ concentration on universal screening would not necessarily indicate $\beta$ thalassaemia.

ه Howard J, et al. Journal of Clinical Pathology 2005;58:556-558. 